\title{
DEVELOPMENT OF DGPS GUIDANCE SYSTEM FOR AGRICULTURAL MACHINERY
}

\author{
Zhijun Meng ${ }^{1}$, Wenqian Huang ${ }^{1}$, Hui Liu ${ }^{2}$, Liping Chen ${ }^{1}$, Weiqiang $\mathrm{Fu}^{1}$ \\ ${ }^{1}$ National Engineering Research Center for Information Technology in Agriculture, Beijing, \\ P. R. China Corresponding author, Email: mengzj@nercita.org.cn \\ ${ }^{2}$ Key Laboratory of Modern Precision Agriculture System Integration Research, Ministry of \\ Education, China Agricultural University, Beijing, 100083
}

Abstract: The purpose of this research was to develop a kind of light bar parallel guidance system based on DGPS for small agriculture machinery. This system consists of field computer, light bar and DGPS. The field computer is developed using PC/104 CPU module, running on which the embedded realtime operation system was customized by a special development tool. Single chip and CAN controller was selected for guidance light bar design, which use a row of indicator LEDs to guide drivers. Field computer and guidance light bar communicate with each other by using CAN bus. The guidance software running on the field computer was designed in object-oriented manner by integrating embedded GIS development kit. Field-testing and experiments have been done to evaluate the accuracy of the system with Trimble AgGPS 132 under different speeds, application width and light bar sensitivity, and the average of offline distance was 0.4517 meter. Results show this kind of guidance system is suitable for small-size machinery and can meet the need for parallel swath guidance for different kinds of field operation.

Keywords: DGPS, Agricultural Machinery, parallel guidance

\section{INTRODUCTION}

Agricultural machinery are usually driven in parallel swath pattern during the operation of seeding, fertilizing, spraying and seedbed preparing, so the machines' moving track will affect the operation directly. In order to avoid overlap or skip in the operation, the scriber, furrow, ditch and crop rows are

Meng, Z., Huang, W., Liu, H., Chen, L. and Fu, W., 2008, in IFIP International Federation for Information Processing, Volume 259; Computer and Computing Technologies in Agriculture, Vol. 2; Daoliang Li; (Boston: Springer), pp. 1307-1310. 
used as guidance in traditional methods. Operators must focus their attention on driving to be sure of the tractor's moving in parallel pattern with the help of this guidance. However, guidance effect is dependent on the proficiency of operators, especially when using a large-scale machine with a wide application width at a high speed (O'Connor, 1996).

GPS guidance and machine vision guidance are the two main promising ways. With the development of precision agriculture, GPS equipments with high accuracy are used widely. Guidance based on GPS and operation path layout has many advantages such as high reliability and less time limitation. Recently, there are many commercial products of Light-bar Guidance System. Researchers do many researches and experiments on the light-bar guidance precision. Buick and Lang researched and analyzed the precision and efficiency between foam marker method and DGPS light-bar guidance system through experiments in 1998 (Buick, 1998).

The purpose of the present study was to develop a kind of light bar parallel guidance system based on DGPS for small agriculture machinery. Field-testing and experiments have been done to evaluate the accuracy of the system with the use of a Trimble AgGPS under different speeds, application width and light bar sensitivity.

\section{SYSTEM DESIGN}

This parallel swathing guidance system consists of field computer, light bar guidance aids and DGPS. Field computer for agriculture machinery is designed for controlling, displaying and data storage during the process of parallel swathing guidance, variable rate application of fertilizer and chemicals, field information collection etc. This field computer is designed based on PCM-3350, ADVANTECH PC/104 CPU module, which with GX1-300 MHz onboard processor and low power, fangless performance. PCM-3350 supports Compact Flash SSD solution, 18-bit TFT LCD, touch panel, some standard communications ports. In order to make field computer support CAN bus communication, PCM 3680 CAN adapter card was selected for this field computer. PCM 3680 support CAN interface memory address of CAN interface can be changed by address switch.

Windows CE.NET was selected as embedded real time operation system (RTOS) for this field computer. Windows CE .NET is 32-bit, real-time, multitasking OS, which is highly componentized, scalable and has wide variety of CPU support. It combines an advanced, real-time operating system with powerful tools for rapidly creating the next generation of smart, connected, and small-footprint devices and Windows CE for Automotive and Industrial Automation. Some configuration and development of Wince 
operation system have been done to meet the field computer hardware environment by using Platform Builder. Platform Builder is a Configuration and debugging tool for deploying an operating system.

In order to avoid overlap or leaving gaps in the field, the light-bar guidance system is used to help the operator to drive the machine moving along the desired parallel passes, which can improve the operation quality and efficiency. W77E58 C51 microcontroller of Winbond Company, the independent CAN controller SJA1000 and CAN transceiver PCA82C250 of Philips Company are adopted, which realize the CAN communication. The high-speed optocouplers $6 \mathrm{~N} 137$ are used for voltage isolation between input and output and watchdog timer X5045P for independent protection for microcontroller. Since the variable rate controller and the light-bar guidance unit are in the same CAN local area network, the guidance unit can receive the deviation data from the controller using the special communication protocol. The 35 LEDs and the LCD can display the deviation in an obvious way. The 74ALS373 and 74F377 are used for address logic and LED control signal output respectively.

In order to meet the need of parallel guidance, the application should provide the functions such as parallel guidance display, vehicle motion control and application data management. This application has to deal with lots of control, operation, object and data. Following object-oriented programming thought, above data and objects concerned in a parallel swath guidance application procedure could be abstracted into different classes. Workspace class was used to manage all of data and objects, which is created when parallel swath guidance application project begins. This object organizes the whole application procedure, creates relevant objects such as logging object, field object, vehicle object and so on. Guidance data communication protocol prescribes the data format for field computer and guidance light bar. Current version of this protocol includes navigation sentence, GPS status sentence, navigation status sentence and machine travel status sentences. This protocol can be extended according to system need. Navigation protocol between the central computer and the stimulation light bar should be defined so as to the navigation message the sender and receiver identify string. A typical navigation string is shown as following: $\$ \mathrm{~L}, \mathrm{~L}, 2, * \mathrm{hh}, \mathrm{CR} / \mathrm{LF}$.

\section{FIELD TEST}

Field test was accomplished in September of 2005 on National Precision Agriculture Demonstration Farm at suburb of Beijing. The vehicle model TD724 was an agricultural tractor made in China, Trimble AgGPS132 DGPS receiver was used for the vehicle positioning. Considering vehicle 
speed, swath width and light bar sensitivity, 9 experiments were conducted totally. There are no ground references for guidance in the testing field. Track points, track line and swath coverage data was recorded during the test. test Recorded data was processed with ArcMap by using layer overlap, intersection and clip tools. A program was designed for getting the distance between the actual track point and the ideal swath. The maximal value of offline distance was 0.6128 meter and the minimal value was 0.3021 meter among the results. And the average offline distance was 0.4517 meter. The field computer and light bar worked well in the test. The guidance system could be proven to have good performance for small-size tractors.

\section{CONCLUSION}

This research designed the parallel swath guidance system based on DGPS for the medium and small size tractors made in China. This system consists of field computer, light bar and DGPS. The field computer is developed using PC/104 CPU module, running on which the embedded realtime operation system was customized by a special development tool. Single chip and CAN controller was selected for guidance light bar design, which use a row of indicator LEDs to guide drivers. Field computer and guidance light bar communicate with each other by using CAN bus. The guidance software running on the field computer was designed in object-oriented manner by integrating embedded GIS development kit. Field test indicated that the guidance system was fit for the small-size tractors. But more tests should be given using RTK-GPS receiver of high precision for reliability and accuracy.

\section{REFERENCES}

Buick, R. White, E. 1998. Comparing GPS guidance with foam marker guidance. In: International Conference on Precision Agriculture. Madison: ASA-CSSA-SSSA, 1035-1045.

O'Connor, M., T. Bell, G. Elkaim, and B. Parkinson. 1996. Automatic steering of farm vehicles using GPS. 3rd International Conference on Precision Agriculture, Minneapolis, MN. 